\title{
VISCOUS DRAG ON A LATERAL MICRO-RESONATOR: FAST 3-D FLUID SIMULATION AND MEASURED DATA
}

\author{
Wenjing Ye \\ School of Mechanical Engineering, Georgia Institute of Technology \\ Atlanta, GA 30339-0405
Xin Wang, Werner Hemmert, Dennis Freeman and Jacob White
EECS, Massachusetts Institute of Technology
Cambridge, MA 02139

\begin{abstract}
Computing drag forces on geometrically complicated 3-D micromachined structures, such as an entire laterally moving comb resonator, is a challenging problem. The standard semi-analytic approach, based on assuming a regular flow patterns such as Couette or one-dimensional Stokes flow, results in a good rough estimate for the drag force but can be insufficiently accurate to correctly predict geometric sensitivities. Designers have not tried to improve the accuracy using numerical simulation, because traditional finite-element based 3-D fluid simulation is too computationally expensive to use for such complicated geometries. However, the recently developed FastStokes solver, based on the precorrected-FFT accelerated iterative methods, has made fluid analysis of an entire comb tractable. Herein, we compare measurements to simulation to show that drag force can be accurately predicted using 3-D simulation. In addition, detailed examination of the results from 3-D simulation indicates that there is significant contribution to drag due to sidewall forces, and therefore structure levitation will not increase quality factor as dramatically as one would expect from Couette flow analysis alone.
\end{abstract}

\section{INTRODUCTION}

Analysis of the dynamic behavior of micromachined devices packaged in the air or fluid requires the determination of damping effects on the devices due to viscous forces from the surrounding fluid. Because of the small spatial scale of the devices, the fluid usually satisfies Stakes' equation. Therefore, computing drag forces involves solving Stokes' equation on geometrically complicated 3-D micromachined structures, such as an entire laterally moving comb resonator, and this is a computationally challenging problem. Instead, semi-analytic approaches are often used, based on assuming a regular flow patterns such as Couette or one-dimensional Stokes flow [1] [2]. These approximations result in a good rough estimate for the drag force but can be insufficiently accurate to correctly predict geometric sensitivities. Designers have not tried to improve the accuracy using numerical simulation, because traditional finite-element based 3 -D fluid simulation is too computationally expensive to use for such complicated geometries. However, the recently developed FastStokes solver [3] [4], based on the precorrected-FFT accelerated iterative methods [5], has made fluid analysis of an entire comb tractable. In this paper, we compare measurements to simulation to show that drag force can be accurately predicted using 3-D simulation. In next section, a short description of FastStokes is given. It is followed by the description of the experimental measurements of the resonant frequency and the quality factor of a micro-resonator. In section 3 , numerical simulations of the drag force and the quality factor of the device are presented. Results are compared with the measured data, and good agreement is demonstrated. In addition, the computational results are examined in detail to show that sidewall forces are significant. Finally, in sections 4 and 5 , we give conclusions and acknowledgements.

\section{FASTSTOKES}

FastStokes is a 3-D fast solver for both steady and unsteady Stokes' equations. The program combines a boundary element method for discretizing an integral formulation of Stokes' equation with precorrected-FFT accerated technique for solving the discretized equations.

The equivalent indirect boundary integral equations for unsteady Stokes' flow in the frequency domain are [6]

$u_{j}\left(\mathbf{x}_{\mathbf{0}}\right)=-\frac{1}{8 \pi \mu} \int_{S} f_{i}(\mathbf{x}) G_{i j}(\hat{\mathbf{x}}) d s$

where $S$ is the surface on the object in an infinite fluid, and the Greens function is given by

$$
\begin{array}{r}
G_{i j}(\hat{\mathbf{x}})=\frac{\delta_{i j}}{r} A(R)+\frac{\hat{x_{i}} \hat{x_{j}}}{r^{3}} B(R) \\
A=2 e^{-R}\left(1+\frac{1}{R}+\frac{1}{R^{2}}\right)-\frac{2}{R^{2}} \\
B=-2 e^{-R}\left(1+\frac{3}{R}+\frac{3}{R^{2}}\right)+\frac{6}{R^{2}} \\
R=\lambda r
\end{array}
$$

In $(1), u_{j}\left(\mathbf{x}_{\mathbf{0}}\right)$ is the $j$ th component of the velocity vector at the source point $\mathbf{x}_{\mathbf{0}}, \mathbf{f}$ is the Stokeslet density function and the real part of $\mathbf{f}$ corresponds to the real part of the surface traction if there is a single moving object that rigidly translates in the fluid, $\rho$ and $\mu$ are the density 
and the viscosity of the fluid respectively, $\hat{x}_{i}$ is the $i$ th component of the relative position vector between the source point and the field point, i.e. $\hat{x}_{i}=x_{0 i}-x_{i}, r$ is the length of the relative position vector $\left(r=\left|\mathbf{x}_{0}-\mathbf{x}\right|\right), \lambda$ is the frequency parameter which is defined as $\lambda^{2}=i \omega \frac{\mu d^{2}}{\rho}$, $\omega$ is the frequency of the fluid and $d$ is the characteristic size of the object.

A piece-wise constant collocation scheme is used to solve (1) [7]. The surface of the object (device) is discretized into $n$ small panels (see, for example, Figure 3 ), and the components of the traction forces are assumed to be constant on each panel. A system of equations for the panel unknowns is then derived by insisting the integral equations are satisfied at each panel centroid. The result is a system which relates the known velocity vector $\mathbf{u}$ to the vector of traction forces $\mathbf{f}$, as in

$$
\left\{\begin{array}{c}
\mathbf{u}^{1} \\
\mathbf{u}^{2} \\
\cdot \\
\cdot \\
\cdot \\
\mathbf{u}^{n}
\end{array}\right\}=P(\omega)\left\{\begin{array}{c}
\mathbf{f}^{1} \\
\mathbf{f}^{2} \\
\cdot \\
\cdot \\
\cdot \\
\mathbf{f}^{n}
\end{array}\right\}
$$

where $\mathbf{u}^{i}$ and $\mathbf{f}^{i}$ are the velocity vector and vector traction force for the $i$ th panel, $P$ is a $3 n \times 3 n$ matrix whose elements are given by

$$
\begin{gathered}
P_{k l}^{i j}(\omega)=\int_{\Delta j}\left[\frac{\delta_{k l} A(R)}{\left|\mathbf{x}^{i}-\mathbf{x}\right|}+\frac{\left(x_{k}^{i}-x_{k}\right)\left(x_{l}^{i}-x_{l}\right)}{\left|\mathbf{x}^{i}-\mathbf{x}\right|^{3}} B(R)\right] d s \\
k, l=1,2,3 .
\end{gathered}
$$

Here, $\mathbf{x}^{i}$ denotes the centroid of the $i$ th panel and $\Delta j$ denotes the surface of the $j$ th panel.

The linear system in (3) could be solved with Gaussian elimination, but then the cost of system solution would grow like the cube of the number of unknowns. For the comb example in Figure 3, there are more than 50,000 unknowns, and Gaussian elimination would require more than 10,000 gigaflops to solve. In addition, storing the matrix grows like the square of the number of unknowns, and for the comb example would require more that 20 gigabytes.

Instead, (3) is solved using a combination of an iterative method, complex GMRES [8], and a precorrectedFFT technique [5]. This combination of techniques results in an algorithm whose solution time and memory grows nearly linearly with the number of panels. For example, the 50,000 unknown problem in Figure 3 can be solved in 15 minutes on a workstation, and requires only one gigabyte of memory.

\section{Experimental Measurement}

The test structure was a surface-micromachined lateral resonator (Figure 1) fabricated using the MUMPS
Table 1: Resonator dimensions

\begin{tabular}{|c|c|}
\hline finger gap & $2.88 \mu \mathrm{m}$ \\
\hline finger length & $39.96 \mu \mathrm{m}$ \\
\hline finger overlap & $19.44 \mu \mathrm{m}$ \\
\hline beam length & $151 \mu \mathrm{m}$ \\
\hline beam width & $1.1 \mu \mathrm{m}$ \\
\hline center plate & $54.9 \mu \mathrm{m} \times 19.26 \mu \mathrm{m}$ \\
\hline thickness & $1.96 \mu \mathrm{m}$ \\
\hline substrate gap & $2 \mu \mathrm{m}$ \\
\hline
\end{tabular}

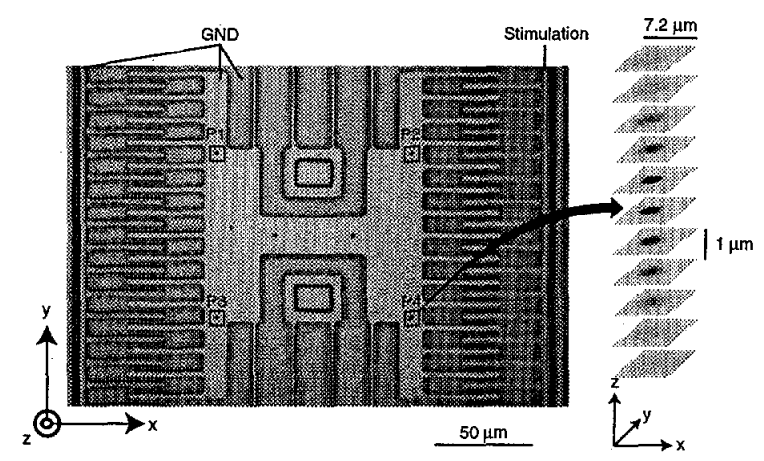

Figure 1: Two- and three-dimensional images of a lateral resonator. The left panel shows the central portion of a lateral resonator imaged near its best plane of focus. The resonator was excited to move by applying an electrical stimulus to the right comb drive while holding the left comb drive and shuttle at ground. Four regions of interest enclosing anti-stiction dimples are indicated by black boxes labeled P1, P2, P3, and P4. A 3D image of an anti-stiction dimple (right panel) is obtained by stacking a sequence of images from multiple planes of focus ( $2 \mu \mathrm{m}$ spacing). The in-plane and out-of-plane scales differ, as shown by the scale bars, for clarity.

process at MCNC (now Cronos Integrated Microsystems Inc., Research Triangle Park, NC). Dimensions are shown in Table (1). The resonator was set into motion in air (atmospheric pressure) using an electrical stimulus to one comb drive. The magnitude and angle of the resulting motions were measured using computer microvision [9] and results are shown in Figure 2. Results were well fit by a second order system response with a resonant frequency of $19.2 \mathrm{kHz}$ and a quality $\mathrm{Q}$ of 27 .

\section{Numerical Simulation}

A second-order spring-rnass-dashpot model described in equation (5) was generated to capture the dynamic benavior of the resonator.

$m_{e f f} \ddot{x}+c \dot{x}+k x=F_{\text {actuator }}$, 

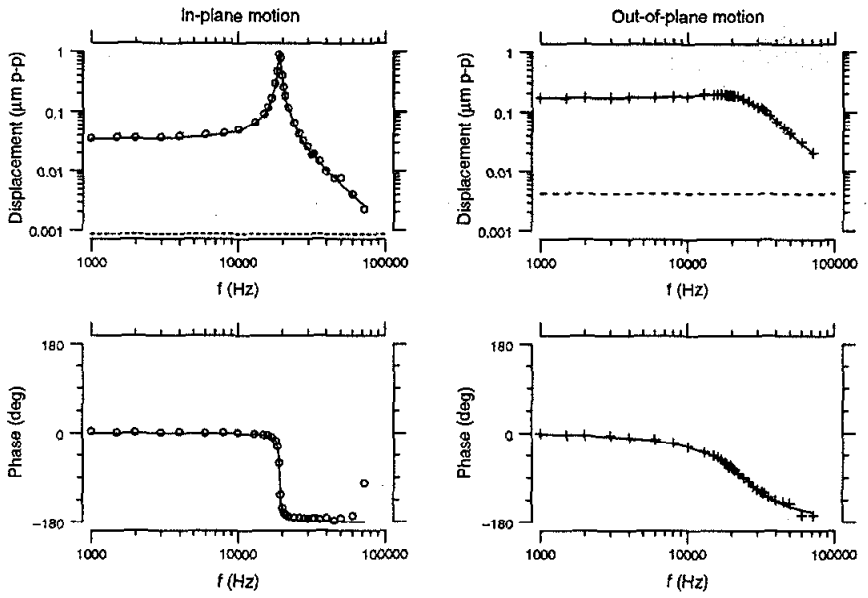

Figure 2: Frequency response for in-plane (left) and outof-plane (right) motion of the anti-stiction dimple. Sinusoidal voltages ( $10 \mathrm{Vpp} A C$ plus $50 \mathrm{~V} \mathrm{DC}$ ) were used to stimulate motions of the resonator. Motions were measured by analyzing a sequence of 3D images (see Figure 1) obtained using stroboscopic illumination at 8 evenly spaced times during the stimulus period. Circles indicate the magnitude and phase of motions measured at 32 frequencies form 1 to $72 \mathrm{kHz}$. Best-fitting second-order resonance curves are shown by the solid lines.

where $x$ is the displacement of the beams and $F_{\text {actuator }}$ is the force given by the comb fingers due to the electric field.

In equation (5), the effective mass $m_{e f f}$ was determined using Rayleigh's method [10]. It was defined as

$m_{e f f}=m_{p}+\frac{1}{4} m_{c}+\frac{12}{35} m_{b}$

where $m_{p}, m_{c}$ and $m_{b}$ are the masses of the center plate, moving comb fingers and the beams respectively. The stiffness of the beams $k$ was computed from the effective mass and measured resonant frequency:

$f=\frac{1}{2 \pi} \sqrt{\frac{k}{m_{e} f f}}$.

For the studied resonator, the effective mass and the stiffness of the spring are $3.5 \times 10^{-11} \mathrm{~kg}$ and $0.511 \frac{\mathrm{N}}{\mathrm{m}}$.

The dashpot damping coefficient was computed by calculating the viscous drag force and then dividing by the velocity. The drag force was computed at the resonant frequency $f=19200 \mathrm{HZ}$. Two models were used: the Couettc flow model (including damping due to both structure-substrate interaction and finger-finger interaction) as in [1], and then the 3-D Stokes model, FastStokes. The surface mesh used for FastStokes is shown in Figure (3). A convergence study was performed to ensure the accuracy of the simulation results. Table (2) shows the simulated drag force on the resonator for different discretizations. The table shows that using 23,424

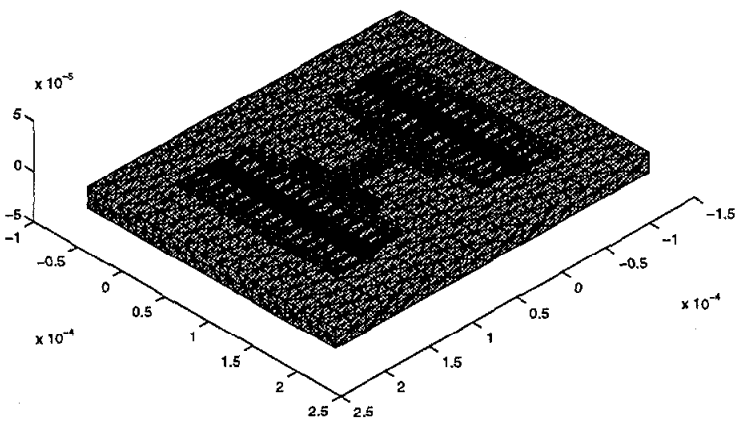

Figure 3: The meshed resonator with substrate, total 16575 panels

Table 2: Viscous drag force on the resonator

\begin{tabular}{|c|c|c|}
\hline Number of Panels & FastStokes & Couette \\
\hline 10048 & $165.04(n N)$ & \multirow[t]{3}{*}{95.02} \\
\hline 16576 & $168.34(n N)$ & \\
\hline 23424 & $169.45(n N)$ & \\
\hline
\end{tabular}

panels results in a drag force that is within one percent of the exact solution to the Stokes' equation.

The quality factor $\mathrm{Q}$ of the resonator was determined from

$Q=\sqrt{\frac{k m_{e f f}}{c}}$.

Results are shown in Table (3), together with the measured Q. As the table shows, the Couette flow model overpredicts the $\mathrm{Q}$ by a factor of nearly two, whereas the FastStoke's based result is within 10 percent. In Figure (4), we show the drag force distribution on the comb. As can be seen from the figure, there are significant contributions to the drag force on the sides of the comb. These contributions are mostly caused by the pressure gradients existing in the flow field and the edge effects due to the finite size of the device. Based on an infinite-plate assumption, both Couette-model and 1-D Stokes-model fail to capture this component of the drag. Integration of the various components of the simulated drag force shows that side forces account for 33 percent of the drag, top force contributes (Stokes-type damping

Table 3: Q factor of the resonator

\begin{tabular}{|l|c|c|c|}
\hline & FastStokes & Couette & Measurement \\
\hline Q factor & 25.16 & 44.59 & 27 \\
\hline
\end{tabular}




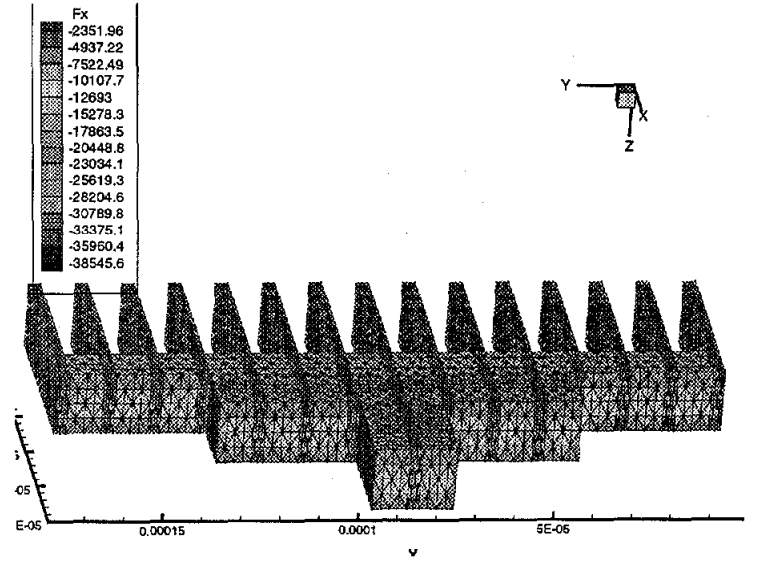

Figure 4: Drag force distribution on the resonator, bottom (substrate-side) view

in the ambient fluid) another 12 percent, and the bottom force (Couette-type damping) is 55 percent. The implication is that levitating the comb will not increase the $\mathrm{Q}$ as much as a Couette flow model predicts.

\section{CONCLUSIONS}

In this paper, we compare measurements to simulation to show that viscous drag force can be accurately predicted using $3-\mathrm{D}$ simulation. A lateral microresonator ocsillating in the air was studied. The drag force was simulated both using FastStokes, a precorrectedFFT accelerated unsteady Stokes solver and a simple Couette flow model. Quality factor of the device was then determined from the simulated results and compared with the experimental data. A good agreement was obtained between the 3 -D results with the experimental data (error is within 10 percent), whereas the Couette model overpredicts the $Q$ by a factor of nearly two. In addition, the detailed examination of the results from 3-D simulation indicates that there is significant contribution to drag due to sidewall forces, and therefore structure levitation will not increase quality factor as dramatically as one would expect from Couette flow analysis alone.

\section{ACKNOWLEDGMENTS}

The authors would like to acknowledge the contributions of Joel Phillips, the author of the precorrectedFFT code used in FastStokes, and Narayan Aluru, the author of the first prototype FastStokes solver. This work was supported by a grant from NSF, and DARPA under the composite CAD program.

\section{REFERENCES}

[1] W. C. Tang, T.-C. H. Nguyen, M. W. Judy and R. T. Howe, "Electrostatic-comb drive of lateral polysil- icon resonators," Sensors and Actuators, vol. A21 23, pp. 328-381, 1990.

[2] Y.-H. Cho, B.-M. Kwak, A. P. Pisano and R. T. Howe, "Viscous energy dissipation in laterally oscillating planar microstructures: a theoretical and experimental study." Proceedings. IEEE. Micro Electro Mechanical Systems. An Investigation of Micro Structures, Sensors, Actuators, Machines and Systems (Cat. No.93CH3265-6). IEEE. pp.93-8. New York, NY, USA, 1993.

[3] N. R. Aluru and J. White, "A Fast Integral Equation Technique for Analysis of Microflow Sensors Based on Drag Force Calculations," Proc. MSM, pp. 283286, Santa Clara, April 1998.

[4] W. Ye, J. Kanapka, X. Wang, J. White, "Efficiency and Accuracy Improvements for FastStokes, A Precorrected-FFT Accelerated 3-D Stokes Solver", Proc. of MSM, pp. 502-505, Puerto Rico, 1999.

[5] J. R. Phillips and J. K. White, "A Precorrected-FFT method for Electrostatic Analysis of Complicated 3D Structures," IEEE Trans. on Computer-Aided Design, October 1997, Vol. 16, No. 10, pp. 1059-1072.

[6] C. Pozrikidis, Boundary integral and singularity methods for linearized viscous flow, Cambridge University Press, Cambridge, 1992.

[7] P. K. Banerjee, The Boundary Element Methods in Engineering, McGraw-Hill Book Company, England, 1981.

[8] Y. Saad and M. Schultz, 'GMRES: A generalized minimal residual algorithm for solving symmetric linear systems,' SIAM J. Sci. Sialis. Comput., 7, 856-869, 1986.

[9] D. M. Freeman, A. J. Aranyosi, M. J. Gordon, and S. S. Hong, "Multidimensional motion analysis of MEMS using computer microvision," 1998 SolidState Sensor and Actuator Workshop, June 8-11, pp. 150-155. 1998.

[10] W. C. Tang, M. G. Lim and R. T. Howe, "Electrostatic comb drive levitation and contral method," Journal of MEMS, vol. 1, No. 4, pp. 170 - 177, 1992. 